\title{
Addressing selective reporting of experiments - the case for predefined exclusion criteria
}

\author{
Kleber Neves and Olavo B. Amaral
}

Institute of Medical Biochemistry Leopoldo de Meis, Federal University of Rio de Janeiro, Brazil

\begin{abstract}
Articles describing experimental data in the life sciences are meant to tell a clear story to the reader. This means that not every experimental attempt ends up published, as failed experiments and uninformative data are typically filtered out by researchers. Freedom to exclude data from an article, however, can lead to reporting bias when exclusion decisions are made after results are in. We discuss how to balance clarity and thoroughness in biomedical research reporting, and suggest that predefined criteria for experimental validity might help in solving this conflict.
\end{abstract}

\section{Introduction}

It is no surprise that science plays a prominent role in the 2018 documentary Behind the Curve (Clark et al., 2018), about the flat-Earth movement in the United States. Interestingly, however, much of the science is performed by the flat-Earthers themselves. One experimentsavvy believer tells of multiple attempts to prove that the Earth is flat by examining the drift of a laser gyroscope over time. His first try, however, falls flat - or round -, as the device shows the $15 \%$ hour drift predicted by a rotating Earth. "We obviously were not willing to accept that", goes that scientist, "and so we started looking for ways to disprove that it was registering the motion of the Earth and that in fact it was registering the motion of the sky". Their next step is to encase the gyroscope in a zero gauss chamber to "shield the energies of the heavens" - which predictably does not prevent the drift. "The next thing we are going to try is encasing the entire apparatus in bismuth. If everything works well l'd like to release it at the conference."

This kind of persistence is likely to be familiar to anyone who has worked in laboratory science. Experiments fail all the time, for both complex and trivial reasons. Because of this, repeating them - typically after tinkering a bit with the protocol - is common, and the initial failed attempts frequently go unreported in published articles. After all, trying to fully present all 
the unsuccessful results of a long, obstacle-prone project might make a paper all but unreadable.

In a recent feature article at elife, Joshua Sanes argues that "in presenting your results, you have to tell the truth and nothing but the truth. What you don't have to do is tell the whole truth. In other words, you can select the results you present them, to shape your narrative" (Sanes, 2019). He is quick to point out the caveat that "if you have results that call your conclusions into question, you need to present them." That, however, was not enough to appease criticism. Commenting on Twitter (e.g. Chambers, 2019, Srivastaja, 2019), open science advocates compared the advice to the "fishing expedition" approach to article writing classically advocated by the psychologist Darryl Bem (2004) - who later became known for his defense of precognition about events that have not yet happened (Bem, 2011).

As laboratory scientists, we understand both sides of the argument: reporting on every experimental failure will increase noise without adding value to the reported results; on the other hand, having unlimited flexibility to decide whether an experiment can be excluded from a paper opens up a huge avenue for bias to creep in - which, when taken to the limit, can lead straight into the flat Earth conference. In this article, we will make our case that predefined inclusion and exclusion criteria for experiments can help solve this conundrum, and discuss ways in which they can be implemented in the workflow of experimental projects.

\section{The many-level file drawer}

Scientific communication is a challenging endeavor. In order to facilitate the understanding of complex ideas and the clear outlining of hypotheses, the scientific article evolved towards a structured format that privileges a well-thought narrative arc meant to tell an intuitive story (Sanes, 2019; Sollaci, 2004). Narrative quality, however, can go against the need for transparency in reporting to ensure reproducibility: as the process of conducting research is vulnerable to a large amount of biases (Chavalarias \& loannidis, 2010, Simmons et al., 2011), selective reporting on what to publish can have severe detrimental consequences on the scientific record (Nosek et al., 2015; Nissen et al., 2016).

Selective reporting can appear at many levels. A considerable body of literature exists on the omission of whole studies, a phenomenon best known as the "file drawer effect" (Rosenthal, 1979). This is best studied in areas such as clinical trials and psychology (e.g. Dwan et al., 2013; Ferguson \& Heene, 2012; Fanelli, 2012), in which meta-analytic statistical methods are 
routinely used for estimating publication bias (Jin et al., 2015). At the level of analysis, there is also evidence of bias in selective reporting of measured outcomes within trials (Williamson et al., 2005). On the other end, closest to data collection, selective post hoc exclusion of data points identified as outliers is a problem that has also been reasonably discussed (Holman et al., 2016).

Selective reporting of experiments is an intermediate level of bias that lies between the omission of studies and that of data points. It appears to be common in scientific fields where a single article typically includes multiple experiments or datasets, as in much of the life sciences. Although this is potentially one of the largest sources of bias in bench research, it has been relatively underdiscussed. The case has been made that the prevalence of significant results within single articles is frequently too high to be credible, considering the statistical power of individual experiments (Schimmack, 2012; Läkens \& Etz, 2017). However, such statistical evidence cannot identify whether this is due to experiments going missing or to bias in the published ones. While one can locate unpublished clinical trials because they are preregistered, or look for mismatching sample sizes in articles to infer removal/loss of subjects, detecting an unreported experiment depends on information typically unavailable to the reader.

Once more, the problem is that making the full information on every experiment conducted within a project might be counterproductive as well. Laboratory science can be technically challenging, and experimental projects hardly ever go well from start to finish; thus, a certain degree of selective reporting can be helpful to separate the wheat from the chaff. Hardly anyone would be interested to know, after all, that your histological sections failed to stain, or that your culture behaved in strange ways because of contamination. Such obvious failures intuitively seem not to belong to the "whole truth" that should be included in an article.

What is the limit, however, to what can be left out of a paper? While most scientists will agree that suppressing results from an article because they do not fit a hypothesis is unethical, few would argue in favor of including every methodological failure in it. The problem, however, arises when one is free to classify experiments into either category after the results are in.

\section{The reverse Texas sharpshooter}

It is all too easy to find something that went wrong with a particular experiment to justify its exclusion. Maybe the controls looked different than before, or one remembers that someone had complained about that particular antibody vial. Maybe the animals seemed very stressed 
that day, or the student who ran the experiment didn't have "a good hand" for surgery. Or maybe an intentional protocol variation apparently made a previously observed effect disappear. This is particularly frequent in exploratory research, where protocols are typically adjusted along the way. It is thus common that people will repeat an experiment again and again with minor tweaks until a certain result is found - frequently one that confirms an intuition or a previous finding (e.g. "I got it the first time, something must have gone wrong this time").

All of the factors above might be sensible reasons to exclude an experiment. However, this makes it all too easy to find a plausible explanation for a result that does not fit one's hypothesis. Confirmation bias can easily lead to discarding experiments that "didn't work" by attributing the results to experimental artifacts. In this case, "not working" conflates negative results - i.e. nonsignificant differences between groups - with methodological failures - i.e. an experiment that is uninterpretable because its outcome was not appropriately measured. Even in the best of intentions, a scientist with too much freedom to explore reasons to exclude an experiment will allow unconscious biases related to its results to influence his decision (Holman et al., 2015).

This problem is analogous to the forking paths in data analysis (Gelman \& Loken, 2013), or the Texas sharpshooter fallacy (Biemann, 2013), in which hypothesizing after the results are known (HARKing) leads a hypothesis to be artificially confirmed by the same data that generated it (Hollenbeck \& Wright, 2017). The only difference is that, while the Texas sharpshooter hits bullseye every time because he draws his target around the point where his bullet landed, the scientist looking to exclude an experiment draws his validation target away from the results - considering the experiment as invalid because of a problem that only becomes critical after the results are seen. Importantly, these decisions - and experiments - will be invisible in the final paper if the norm is to set aside the pieces that do not fit the story (Sanes, 2019).

\section{Preregistration in confirmatory research}

One much-discussed solution to the problem of analysis flexibility is preregistration of hypotheses and methods (Forstmeier et al., 2017; Nosek et al., 2018). The practice is still mostly limited to areas such as clinical trials (in which registration is mandatory in many countries) and psychology (in which the movement has gained traction over reproducibility concerns), and is not easy to implement in laboratory science, where protocols are frequently decided along the 
course of a project. Nevertheless, although exploratory science might be the backbone of most basic science projects, a confirmation step with preregistered methods could greatly improve the validation of published findings (Kimmelman et al., 2014; Mogil \& Macleod, 2017).

Interestingly, the concept of preregistration could also be applied to criteria to determine whether an experiment is methodologically sound or not, and thus amenable to suppression from a published paper. Scientists are already used to including controls to assess the internal validity of their methods. In PCR experiments, for instance, measures are typically taken along the way to alert the researcher when something goes wrong: the ratio of absorbance of an RNA sample at 280 and $260 \mathrm{~nm}$ is used as a purity test for the sample, and non-template controls are typically used to check for specificity of amplification (Matlock, n.d.).

Rarely, however, are criteria for what constitutes an appropriate result for a positive or negative control decided and registered in advance, leading the researcher to make this decision once the results of the experiment are in. This not only fails to prevent bias, but actually adds degrees of freedom: much like adding variables or analysis options, adding methodological controls can provide the researcher with more possible justifications to exclude experiments (Wicherts et al., 2016). Once more, the solution seems to require that the criteria to discard an experiment based on these controls be set in advance to counter the possibility of bias.

One may argue that preregistering exclusion criteria is unnecessary if the data for all experiments performed are presented with no censoring (Oberauer \& Lewandowsky, 2019). In this setting, it is up to the reader to judge whether the entire set of data fits a hypothesis, as performing reanalyses of the data - or the entire set of possible analyses (Steegen et al., 2016) can show how much the conclusions depend on certain decisions, such as ignoring an experiment. However, this can only happen if all collected data are indeed presented - which can go against the need to convey information in a narrative form (Sanes, 2019). If some form of data filtering - for failed experiments, noisy signals or uninformative data - is important for clarity (and this may very well be the case) the only way to prevent bias is by setting exclusion criteria beforehand.

\section{Prespecified criteria to clean up the data record}

A solution to selective reporting, thus, is to set prespecified criteria to consider an experiment as valid or not - or a set of data as relevant or not for analysis - before it is performed/collected. These include inclusion and exclusion criteria for animals or cultures to be 
used, positive and negative controls to determine if an assay is sensitive and/or specific, and additional variables or experiments to verify that an intervention has worked as expected. Ideally, these criteria should be as objective as possible, with thresholds and rules for when data must be included and when it should be discarded. Importantly, when criteria for validity are met, this should be taken as evidence that the experiment is appropriate, and that it would thus be unethical to exclude it from an article reporting on the data.

As for any decision involving predefined thresholds, concerns over sensitivity and specificity arise: criteria that are too loose might lead to the inclusion of questionable or irrelevant data on a paper, whereas those that are too stringent could lead meaningful experiments to be discarded. As with preregistration or statistical significance thresholds, this should not discourage researchers from addressing these limitations in an exploratory manner one is always free to show data that does not fit validity criteria if this is clearly pointed out. What is important is that authors are transparent about it - and that the reader knows whether they are following prespecified criteria to ignore an experiment or have decided on it after seeing the results (Hollenbeck \& Wright, 2017). Importantly, this can only happen when data is shown - meaning that decisions to ignore an experiment with no predefined reason should inevitably be discussed alongside its results.

As coordinators of the Brazilian Reproducibility Initiative (Amaral et al., 2019), a multicenter replication of 60-100 experiments of the Brazilian biomedical literature over the last 20 years conducted by a team of more than 60 labs, we have been faced with the need for validation criteria in many stages during protocol development. As the project is meant to be confirmatory in nature, we intend to preregister every protocol, including the analysis plan. Furthermore, to make sure that each replication is methodologically sound, we are stimulating laboratories to add as many additional controls as they judge necessary to each experiment. To deal with the problem raised in this essay, however, we are also requiring that they prespecify their criteria for using the data from these controls in the analysis.

For RT-PCR experiments, for instance, controls for RNA integrity and purity must be accompanied by which ratios will allow inclusion of the sample in the final experiment - or, conversely, will lead data to be discarded. For cell viability experiments using the MTT assay, positive controls for cell toxicity are desirable to test the assay's sensitivity, but must include thresholds for inclusion of the experiment (e.g. a reduction of at least $\mathrm{X} \%$ in cell viability in the positive control). For behavioral experiments, accessory measurements to evaluate an 
intervention's known effects (e.g. weight in the case of high-calorie diets) can be used to confirm that it has worked as expected, and that testing its effects on other variables is warranted. Once more, thresholds must be set beforehand, and failure to meet inclusion criteria will lead the experiment to be considered invalid and repeated in order to attain a usable result.

This has not been an easy exercise, as even though researchers are quite apt at suggesting controls, they are not used to setting objective criteria for them to be used when analyzing data. However, in a pre-registered, confirmatory project, we feel that this is vital to allow us to adjudicate on whether failure to replicate a result constitutes a contradiction of the original finding or a methodological artifact. As one cannot anticipate all possible problems, it is likely that, in some experiments at least, such prespecified criteria might turn out not be ideal in separating successful and failed experiments. Nevertheless, for the sake of transparency, we feel that it is important that any post-hoc decisions for considering experiments as unreliable are marked as so, and that both the decision and its impact on the results are fully disclosed. Once more, there is no inherent problem with exploratory research or data-dependent choices; the problem is when these are done secretly and communicated selectively (Hollenbeck \& Wright, 2017; Simmons et al., 2011).

\section{Conclusion}

Although we have focused on inclusion and exclusion criteria for deciding on whether or not including experiments in an article, the idea of prespecified criteria can meaningfully apply to any field in which some degree of data censoring is important. Fields in which data must be filtered or selected before analysis, such as electrophysiology, functional neuroimaging or epidemiology, are subject to many researcher degrees of freedom in preprocessing pipelines (Phillips, 2004; Carp, 2012; Botvinik-Nezer et al., 2019); thus, prespecification of criteria for these steps is likely warranted to prevent bias. Genomics and other high-throughput fields have also evolved towards standard evaluation criteria to avoid ad-hoc analysis or simple thresholding in selecting experimental hits for further analysis (Kang et al., 2012). This suggests that communities centering on specific methods can find consensus on which criteria are minimally necessary to draw the line between data that can be censored and those that must be analyzed.

Such changes will only happen on a larger scale, however, if researchers are aware of the potential impacts of post hoc decisions on the reliability of results, an area where the life 
sciences still lag behind other fields. Rather than waiting, however, individual researchers focusing on transparency and reproducibility should consider the possibility of setting - and ideally registering -predefined inclusion and exclusion criteria for experiments in their protocols. As Richard Feynman once said, "science is a way of trying not to fool yourself, and you are the easiest person to fool" (Feynman, 1974) - something that is all too obvious in the flat-Earth approach to dealing with "failed" experiments. On the bright side, you can also be a good advisor on whether you are making a fool of yourself to prove a theory - but not when you are in the process of doing it. The easiest way to make this advice heard, thus, is to explicitly state what an appropriate experiment means before starting it, and to stick to your view after the results are in.

\section{References}

Amaral, O. B., Neves, K., Wasilewska-Sampaio, A. P., \& Carneiro, C. F. (2019). The Brazilian Reproducibility Initiative. eLife, 8, e41602. https://doi.org/10.7554/eLife.41602

Bem, D. J. (2004). Writing the empirical journal article. In J. M. Darley, M. P. Zanna, \& H. L. Roediger III (Eds.), The compleat academic: A career guide (p. 185-219). American Psychological Association.

Bem, D.J. (2011) Feeling the future: Experimental evidence for anomalous retroactive influences on cognition and affect. Journal of Personality and Social Psychology 100(3), 407-425. https://doi.org/10.1037/a002152

Biemann, T. (2013) What if we were Texas sharpshooters? Predictor reporting bias in regression analysis. Organizational Research Methods 16(2), 335-363. https://doi.org/10.1177/1094428113485135

Botvinik-Nezer, R., Holzmeister, F. Camerer, C.F., Dreber, A., Huber, J., Johanesson, M, ... Schonberg, $T$ (2019). Variability in the analysis of a single neuroimaging dataset by many teams. bioRxiv, https://doi.org/10.1101/843193

Carp, J. (2012) On the plurality of (methodological) worlds: estimating the analytic flexibility of fMRI experiments. Frontiers in Neuroscience 6, 149. https://doi.org/10.3389/fnins.2012.00149.

Chambers C. (2019). https://twitter.com/chrisdc77/status/1159131460504305664?s=20 (accessed Dec. 11, 2019).

Chavalarias, D., loannidis, JP (2010). Science mapping analysis characterizes 235 biases in biomedical research. Journal of Clinical Epidemiology 63(11), 1205-15. https://doi.org/10.1016/j.jclinepi.2009.12.011.

Clark, C. (Producer), Andert, N. (Producer) \& Clark, D.J. (Producer/Director). (2018). Behind the Curve [Motion Picture]. United States: Detla-v Productions.

Dwan K, Gamble C, Williamson PR, Kirkham JJ, Reporting Bias Group. 2013. Systematic review of the empirical evidence of study publication bias and outcome reporting bias - An updated review. PLOS ONE. 8(7): e66844. https://doi.org/10.1371/journal.pone.0066844 
Fanelli, D. (2012). Negative results are disappearing from most disciplines and countries. Scientometrics, 90(3), 891-904. https://doi.org/10.1007/s11192-011-0494-7

Ferguson, C. J., \& Heene, M. (2012). A Vast Graveyard of Undead Theories: Publication Bias and Psychological Science's Aversion to the Null. Perspectives on Psychological Science, 7(6), 555-561. https://doi.org/10.1177/1745691612459059

Feynman, R. (1974). Cargo cult science. http://calteches.library.caltech.edu/51/2/CargoCult.htm (accessed Nov. 28, 2019)

Forstmeier, W., Wagenmakers, E.-J., \& Parker, T. H. (2017). Detecting and avoiding likely falsepositive findings - a practical guide: Avoiding false-positive findings. Biological Reviews, 92(4), 1941-1968. https://doi.org/10.1111/brv.12315

Gelman, A. (2017). Honesty and Transparency are Not Enough. Chance. Retrieved from http://www.stat.columbia.edu/ gelman/research/published/ChanceEthics14.pdf

Gelman, A., \& Loken, E. (2013). The garden of forking paths: Why multiple comparisons can be a problem, even when there is no 'fishing expedition'or 'p-hacking'and the research hypothesis was posited ahead of time. Downloaded January, 30, 2014.

Hollenbeck, J. R., \& Wright, P. M. (2017). Harking, Sharking, and Tharking: Making the Case for Post Hoc Analysis of Scientific Data. Journal of Management, 43(1), 5-18. https://doi.org/10.1177/0149206316679487

Holman, L., Head, M. L., Lanfear, R., \& Jennions, M. D. (2015). Evidence of experimental bias in the life sciences: why we need blind data recording. PLoS Biology, 13(7), e1002190.

Holman, C., Piper, S. K., Grittner, U., Diamantaras, A. A., Kimmelman, J., Siegerink, B., \& Dirnagl, U. (2016). Where Have All the Rodents Gone? The Effects of Attrition in Experimental Research on Cancer and Stroke. PLOS Biology, 14(1), e1002331. https://doi.org/10.1371/journal.pbio.1002331

Jin, Z.C., Zhou, X.H., He, J (2015). Statistical methods for dealing with publication bias in metaanalysis. Statistics in Medicine, 34(2), 343-60. https://doi.org/10.1002/sim.6342.

Kang, D. D., Sibille, E., Kaminski, N., \& Tseng, G. C. (2012). MetaQC: Objective quality control and inclusion/exclusion criteria for genomic meta-analysis. Nucleic Acids Research, 40(2), e15e15. https://doi.org/10.1093/nar/gkr1071

Kilkenny, C., Browne, W. J., Cuthill, I. C., Emerson, M., \& Altman, D. G. (2010). Improving Bioscience Research Reporting: The ARRIVE Guidelines for Reporting Animal Research. PLoS Biology, 8(6), e1000412. https://doi.org/10.1371/journal.pbio.1000412

Kimmelman, J., Mogil, J.S., Dirnagl, U. (2014) Distinguishing between exploratory and confirmatory preclinical research will improve translation. PLoS Biol 12(5): e1001863. https://doi.org/10.1371/journal.pbio.1001863.

Lakens, D., \& Etz, A. J. (2017). Too true to be bad: When sets of studies with significant and nonsignificant findings are probably true. Social Psychological and Personality Science, 8(8), 875-881.

Matlock, B. (n.d.). Assessment of Nucleic Acid Purity. Retrieved September 20, 2019, from https://assets.thermofisher.com/TFS-Assets/CAD/Product-Bulletins/TN52646-EO215M-NucleicAcid.pdf

Mogil, J.S., Macleod, M.R. (2017). No publication without confirmation. Nature 542(7642), 409411, https://doi.org/10.1038/542409a

Nissen, S. B., Magidson, T., Gross, K., \& Bergstrom, C. T. (2016). Publication bias and the canonization of false facts. Elife, 5 , e21451. 
Nosek, B. A., Alter, G., Banks, G. C., Borsboom, D., Bowman, S. D., Breckler, S. J., ... Yarkoni, T. (2015). Promoting an open research culture. Science, 348(6242), 1422-1425. https://doi.org/10.1126/science.aab2374

Nosek, B.A., Ebersole, C.R., DeHaven, A.C., Mellor, D.T. (2018). The preregistration revolution. Proceedings of the National Academy of Science of the United States of America, 115(11), 2600-2606. https://doi.org/10.1073/pnas.1708274114

Oberauer, K., Lewandowsky, S. (2019). Addressing the theory crisis in psychology. Psychonomic Bulletin \& Review, 26(5), 1596-1618. https://doi.org/10.3758/s13423-019-01645-2

Phillips, C. (2004) Publication bias in situ. BMC Medical Research, 4, 20. http://doi.org/10.1186/1471-2288-4-20

Piletič, K., \& Kunej, T. (2017). Minimal Standards for Reporting microRNA:Target Interactions. OMICS: A Journal of Integrative Biology, 21(4), 197-206. https://doi.org/10.1089/omi.2017.0023

Reimer, C. B., Chen, Z., Bundt, C., Eben, C., London, R. E., \& Vardanian, S. (2019). Open Up - the Mission Statement of the Control of Impulsive Action (Ctrl-ImpAct) Lab on Open Science. Psychologica Belgica, 59(1), 321. https://doi.org/10.5334/pb.494

Rosenthal, R. (1979). The file drawer problem and tolerance for null results. Psychological Bulletin, 86(3), 638-641.

Sanes, J. R. (2019). Tell me a story. ELife, 8, e50527. https://doi.org/10.7554/eLife.50527

Schimmack, U. (2012). The ironic effect of significant results on the credibility of multiple-study articles. Psychological Methods, 17(4), 551-566. https://doi.org/10.1037/a0029487

Simmons, J. P., Nelson, L. D., \& Simonsohn, U. (2011). False-positive psychology: Undisclosed flexibility in data collection and analysis allows presenting anything as significant. Psychol. Sci., 22(11), 1359-1366. https://doi.org/10.1177/0956797611417632

Srivastaja, S. https://twitter.com/hardsci/status/1159134233375285248 (accessed Dec. 11, 2019).

Steegen, S., Tuerlinckx, F., Gelman, A., Vanpaemel, W (2016). Increasing transparency through a multiverse analysis. Perspectives on Psychological Science. 11(5), 702 -712

Sollaci, L. B. (2004). The introduction, methods, results, and discussion (IMRAD) structure: A fifty-year survey. J Med Libr Assoc, 4.

Wicherts, J. M., Veldkamp, C. L. S., Augusteijn, H. E. M., Bakker, M., van Aert, R. C. M., \& van Assen, M. A. L. M. (2016). Degrees of Freedom in Planning, Running, Analyzing, and Reporting Psychological Studies: A Checklist to Avoid p-Hacking. Frontiers in Psychology, 7. https:// doi.org/10.3389/fpsyg.2016.01832

Williamson, P. R., Gamble, C., Altman, D. G., \& Hutton, J. L. (2005). Outcome selection bias in meta-analysis. Statistical Methods in Medical Research, 14(5), 515-524. https://doi.org/10.1191/0962280205sm415oa 